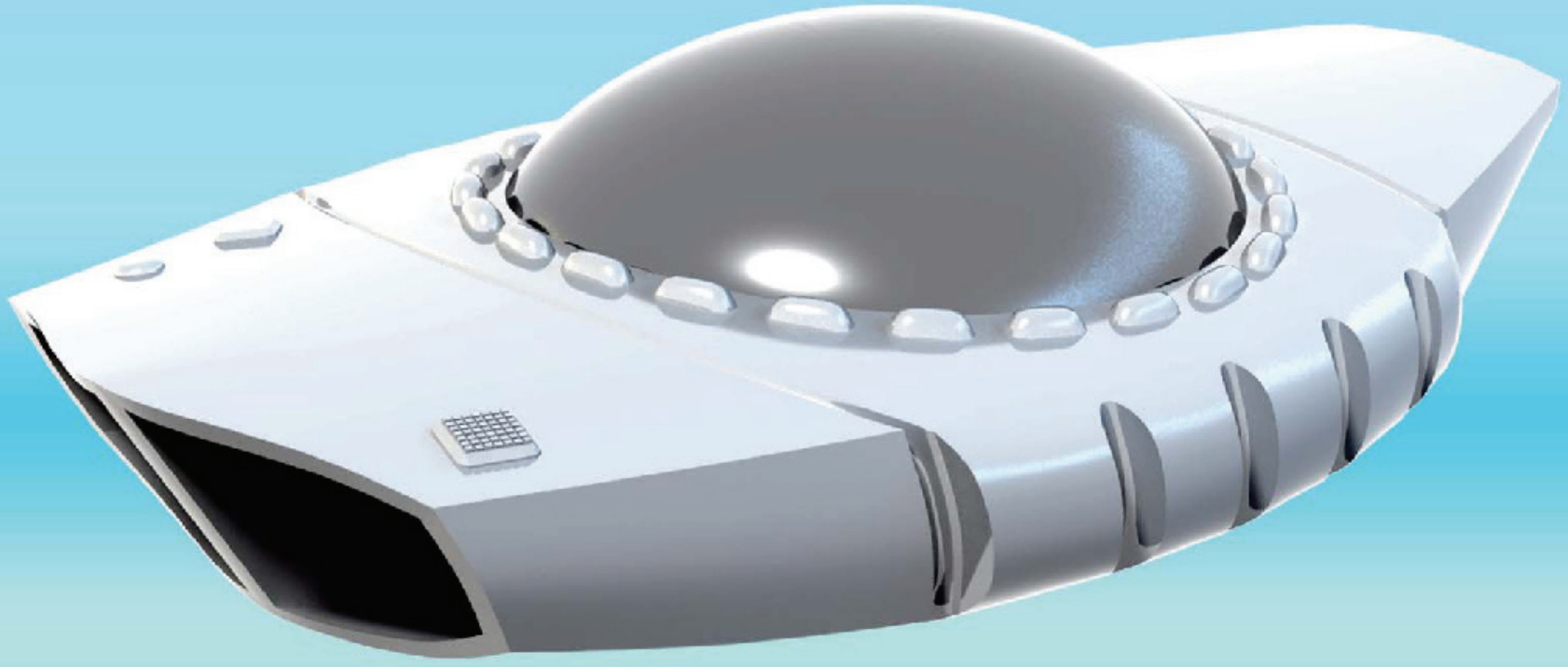

Prof. Dr.-Ing. Christian Karnutsch

Institut für Optofluidik und integrierte nanophotonische Systeme (IONAS), Hochschule Karlsruhe

\title{
Mini-U-Boot in der menschlichen Blutbahn
}

Schon lange liefert die Vision, eine Art Mini-U-Boot in den menschlichen Körper zu injizieren, Inspiration für abenteuerliche und faszinierende Geschichten. Erforscht und entwickelt wird ein derartiges U-Boot mit dem Namen „Nautilos“ am Institut für Optofluidik und integrierte Nanophotonik der Hochschule Karlsruhe.

\section{Ein möglicher Paradigmenwechsel}

Ideal wäre es, Krebszellen schon sehr früh in ihrer Entstehung zu entdecken und diese an der Vermehrung und Ausbreitung zu hindern. Diese Überlegung führte Prof. Karnutsch dazu, das Projekt "Nautilos" (Nearly Autonomous InjectabLe Optofluidic System) ins Leben zu rufen. Der Ansatz versucht biomedizinische Sensorsysteme zu entwickeln, zu verbessern und vor allem zu miniaturisieren. Grundgedanke ist, ein aktiv angetriebenes Mini-U-Boot - eine Art Kapsel - in die menschliche Blutbahn zu injizieren, das dort kontinuierlich das Blut nach Krebszellen absucht und diese gezielt zerstört. Dazu benötigt „Nautilos" drei Hauptkomponenten:

- einen Antriebs- und Kommunikationsstrang,

- eine Diagnoseeinheit für die Untersuchung des Blutes. Hier soll die moderne Technologie der Optofluidik zum Einsatz kommen, eine Kombination von Flüssigkeitstransport und optischen Untersuchungsmethoden auf kleinstem Maßstab sowie

- ein therapeutisches System. Dies kann ein Medikament sein, das lokal appliziert wird, oder ein Nanolaser, der gezielt einzelne Zellen durch Wärme zerstört.
Was so einfach klingt, stößt bei der Umsetzung in die Praxis an schier unüberwindliche Hürden. Zum Einen muss ein hoch komplexes technisches Gebilde extrem stark miniaturisiert werden: „Nautilos“ kann max. einen Durchmesser von $500 \mu \mathrm{m}$ bei 1-2 mm Länge haben. Zum Anderen muss ein Fremdkörper im menschlichen Körper dauerhaft beweglich bleiben, und es muss sichergestellt sein, dass „Nautilos" nicht eine Blutbahn blockieren und dabei große Schäden anrichten kann.

\section{Herausforderung: Diagnoseeinheit}

Die größte Herausforderung ist jedoch die biomedizinische Diagnoseeinheit. Sie existiert noch nicht. Nach welchem Prinzip soll sie funktionieren? Sie könnte z. B. nach Tumormarkern Ausschau halten? Aber was ist die Schlussfolgerung aus dem Ganzen? "Nautilos" ist eine Chance, neue Gedankenprozesse in Gang zu setzen und einen Paradigmenwechsel anzustreben. Weg vom Zerstören bereits entstandener Tumore, hin zur Prävention und zur Verhinderung der Krebsentstehung.

Die heutigen Voraussetzungen lassen darauf hoffen, in etwa 20 bis 25 Jahren das erste einsatzfähige Mini-U-Boot zur Krebsbekämpfung in der menschlichen Blutbahn herstellen zu können. Essentiell dafür ist der interdisziplinäre Dialog zwischen Ingenieuren, Medizinern, Biologen und Chemikern. Und vielleicht sind solche Projekte auch ein Anstoß, hochgradig interdisziplinär angelegte Forschungszentren ins Leben zu rufen. Mehr Informationen zum Thema "Nautilos" sowie Vorträge, Konferenzen und Workshops sind auf der Internetseite www.ionas.eu zu finden. 